\title{
Tratamiento actual del cáncer de próstata de alto riesgo y localmente avanzado
}

\author{
Esquena Fernández S*, Maroto Rey P**, Sancho Pardo G***, Palou Redorta J*, \\ Villavicencio Mavrich $\mathrm{H}^{*}$. \\ *Servicio de Urología, Fundació Puigvert. **Servicios de Oncología y ${ }^{* * *}$ Radioterapia. Hospital Sant Pau. Barcelona. \\ Actas Urol Esp. 2007;31(5):445-451
}

\section{RESUMEN}

TRATAMIENTO ACTUAL DEL CÁNCER DE PRÓSTATA DE ALTO RIESGO Y LOCALMENTE AVANZADO

El tratamiento del cáncer de próstata localmente avanzado continúa siendo controvertido. Las opciones terapéuticas comprenden desde la prostatectomía radical (PR), a la radioterapia (RT) y la hormonoterapia (HT). Se ha efectuado una revisión en la base de datos Medline de los trabajos publicados en los últimos 15 años, con las palabras clave en inglés: "cáncer de próstata", "localmente avanzado", "alto riesgo" y "tratamiento". Cincuenta y uno de 329 artículos fueron seleccionados y revisados. El criterio de selección incluía un mínimo nivel de evidencia científica IIa, destacando alguna referencia puntual con evidencia IV. Como demuestran múltiples estudios aleatorios, estos pacientes se pueden beneficiar de una terapia combinada con RT y HT. La PR en enfermedad localmente avanzada ha demostrado su utilidad en algunos casos seleccionados. Los resultados a largo plazo de algunas series son equiparables a los obtenidos con RT y HT. Además, la posibilidad de sobreestadiaje clínico también es un argumento a favor de la PR. Se realiza una revisión actualizada de todas las posibles opciones disponibles en el tratamiento de estos tumores.

Palabras clave: Cáncer de próstata. Localmente avanzado. Alto riesgo. Tratamiento.

\section{ABSTRACT}

\section{CURRENT TREATMENT IN HIGH RISK AND LOCALLY ADVANCED PROSTATE CANCER}

Treatment of locally advanced prostate cancer remains controversial. Treatment options include radical prostatectomy (PR), radiotherapy (RT) and hormonotherapy (HT). A Medline database search with key words "prostate cancer", "locally advanced", "high risk" and "treatment" in articles published during the last 15 years was done. Fifty one out of 329 papers were selected and reviewed. Selection criteria were a minimum of scientific evidence level of IIa, except for some specific level IV reference. Numerous randomized studies show that patients may benefit of a combined therapy with RT and HT. RP has shown its usefulness in selected cases of locally advanced prostate cancer. Results of long follow-up series are similar to those obtained with RT and HT. Furthermore, the possibility of clinical over staging is an argument in favour of RP. We perform an updated revision of every possible choice available in the treatment of these tumours.

Keywords: Prostate cancer. Locally advanced. High risk. Treatment.

$\mathrm{L}$ a experiencia acumulada en el tratamiento del carcinoma de próstata localizado es extensa, tanto en su vertiente del manejo quirúrgico como en su vertiente de tratamiento con radioterapia. El análisis de los pacientes tratados ha permitido el desarrollo de tablas predictivas de control de la enfermedad que permiten individualizar la opción terapéutica en cada paciente. En el cáncer de próstata localmente avanzado no existe un tratamiento estándar consensuado, por lo que revisaremos las diferentes opciones de tratamiento contempladas en la actualidad.

Se ha efectuado una revisión en la base de datos Medline de los trabajos publicados en los últimos 15 años, con las palabras clave en inglés: "cáncer de próstata", "localmente avanzado", "alto riesgo” y 
"tratamiento", obteniendo 329 artículos, seleccionando 51 que fueron revisados en profundidad. El criterio de selección incluía un mínimo nivel de evidencia científica IIa, destacando alguna referencia puntual con evidencia IV. Con esta revisión se realiza una actualización de todas las posibles opciones disponibles en el tratamiento de estos tumores.

\section{CONCEPTOS DE CARCINOMA DE PRÓSTATA LOCALMENTE AVANZADO Y DE ALTO RIESGO}

Se consideran como tumores de alto riesgo a aquellos que aunque aparentemente localizados tienen un gran riesgo de presentar recidiva local o a distancia. Tradicionalmente se ha aplicado el término de carcinoma localmente avanzado de próstata a toda lesión palpable (y/o visible por técnicas de imagen) que sobrepasa la cápsula prostática y/o infiltra las vesículas. De acuerdo con el riesgo de recidiva la National Comprehensive Cancer Network $(\mathrm{NCCN})^{1}$ distingue entre un tumor de alto riesgo (estadio T3a ó Gleason 8-10 ó PSA > $20 \mathrm{ng} / \mathrm{ml}$ ) y un tumor de muy alto riesgo (combinación de las tres premisas previas o los estadios T3b-T4). En el tumor de alto riesgo las opciones terapéuticas serían prostatectomía radical (PR) con linfadenectomía o bien radioterapia (RT) conformada tridimensional (RTC-3D) en combinación con hormonoterapia (HT). En el tumor de muy alto riesgo se considera la RTC-3D con HT como tratamiento de elección.

Los objetivos del tratamiento en el carcinoma de alto riesgo son:

a. Curación.

b. Control de la progresión local del tumor.

c. Aumento del intervalo libre de metástasis.

d. Mejora de la calidad de vida.

A pesar de la llamada "migración de estadio clínico" que ha sufrido el cáncer de próstata desde que se realiza el cribaje mediante PSA, la importancia de los tumores de alto riesgo no ha disminuido porque estos pacientes tienen peor pronóstico que aquellos con enfermedad clínicamente órgano-confinada, y representan aproximadamente el 10\% de hombres que reciben un diagnóstico inicial de cáncer de próstata ${ }^{2,3}$. Por tanto, el control local del tumor es particularmente importante en estos pacientes por el riesgo de progresión sintomática.
Antes de la comercialización del test de PSA sérico (Era pre-PSA), los factores pronóstico más importantes en el carcinoma de próstata eran el estadio y el grado. El fracaso del tratamiento local se definía por criterios clínico-radiológicos. Con RT externa, los pacientes con cáncer localmente avanzado (T3/T4) alcanzaban unas tasas de control local a 5 y 10 años del 88\% y 81\%, una supervivencia libre de enfermedad del 59\% y $46 \%$, y una supervivencia global del 72\% y 47\%, respectivamente. $\mathrm{Si}$ bien la supervivencia de estos pacientes estaba afectada de forma incuestionable por otros factores como el Gleason, que determinaba la presencia de micrometástasis, la incidencia de recidiva local detectada clínicamente era del $40 \%$ a 10 años $^{4}$. La eficacia de la RT empieza a cuestionarse cuando aparecen estudios en los que se valoran los resultados en función del control bioquímico, obteniéndose altos porcentajes de fallo bioquímico (supervivencia libre de fallo bioquímico de $47 \%$ y $18 \%$ a 5 y 10 años respectivamente) con la administración de dosis medias de $68.4 \mathrm{~Gy}^{5}$. El valor del PSA surge como un factor pronóstico indiscutible conjuntamente con el estadio y el grado de Gleason.

A continuación presentamos las distintas opciones de tratamiento contempladas para estos tumores localmente avanzados y de alto riesgo, así como sus posibles indicaciones en cada caso.

\section{PROSTATECTOMÍA RADICAL}

\section{Tumores cT3a Gleason $<8$ y PSA $<20$ ng/ml}

Hasta hace poco tiempo, la cirugía radical fue frecuentemente evitada, debido a la alta tasa de enfermedad extracapsular y a la inquietud que la PR podría no ser curativa en la mayoría de casos. Pero los resultados de varias series recientes de PR sugieren que podría ser una buena opción de tratamiento. La guía clínica de cáncer de próstata de la Asociación Europea de Urología (EUA) actualmente contempla a la PR como una opción en pacientes seleccionados con tumores cT3 pequeños y unilaterales, con un PSA menor de 20 $\mathrm{ng} / \mathrm{mL}$, una puntuación de Gleason menor de 8 y una expectativa de vida mayor de 10 años ${ }^{6}$, obteniendo una morbilidad parecida a la misma técnica realizada en enfermedad localizada y unos resultados equiparables a los obtenidos con RT más HT. En 2005 la Mayo Clinic publicó datos 
mostrando que la PR en el estadio cT3, administrando en algunos casos HT adyuvante, tenía una supervivencia libre de progresión y cáncer específica a los 5, 10 y 15 años del 85\%, 73\% y 67\%, y del 95\%, 90\% y 79\%, respectivamente, y una supervivencia libre de fallo bioquímico a los 10 años del $43 \%$, por tanto claramente competitiva con los resultados del tratamiento mediante la RT y la $\mathrm{HT}^{7}$. De forma similar, una serie combinada de Baylor y Memorial Sloan-Kettering de 176 pacientes cT3 de un total de 5.182 pacientes tratados mediante $\mathrm{PR}$, ha mostrado una tasa de supervivencia libre de enfermedad a los 10 años del $44 \%$, y sólo el $11 \%$ de los hombres habían muerto de cáncer de próstata con una media de seguimiento de 6,4 años ${ }^{8}$. Finalmente, en el estudio 8894 del SouthWest Oncology Group, se realizó una comparación randomizada de orquiectomía y orquiectomía más flutamida en el tratamiento de cánceres de próstata metastáticos, y los pacientes que tenían mejor respuesta a la HT y mayor supervivencia, fueron los tratados mediante PR antes del desarrollo de la enfermedad metastásica ${ }^{9}$.

Recientes publicaciones demuestran que la morbilidad perioperatoria por PR en pacientes con enfermedad cT3 es similar a la previamente presentada en series anteriores de PR en cT2. Además, no informan de una mayor dificultad técnica para la realización de la intervención comparando con estadios inferiores ${ }^{5,10}$.

\section{Tumores localizados con Gleason $>8$}

Aunque muchos trabajos han afirmado que un Gleason $\geq 8$ es factor pronóstico de recurrencia y enfermedad diseminada ${ }^{12,13}$, algunos estudios postulan que la $\mathrm{PR}$ en pacientes con Gleason $\geq 8$ consigue una supervivencia libre de recidiva bioquímica similar a la RT a los 5 y 8 años, pero con una menor tasa de recidiva local sin llegar a ser significativo (6\% vs $12 \%)^{14}$.

\section{Tumores clinicamente sobreestadiados}

Un grupo de pacientes que aparecen claramente beneficiados del tratamiento mediante PR son los tumores sobreestadiados en estadio cT3, donde al obtener la anatomía patológica de las piezas quirúrgicas se demuestran como cánceres órganoconfinados (pT2), y por tanto, potencial- mente curables sólo con cirugía. Sin embargo este sobreestadiaje varia según las series publicadas entre un $10 \%$ y un $30 \% \%^{7,13,15-17}$, mientras que sólo entre un 5\% y un 8\% de los tumores cT3 estarían infraestadiados (pT4) ${ }^{12,16,17}$. Por esta razón, en todos los pacientes con cáncer de próstata, pero sobretodo en este grupo, la realización de un aproximado estadiaje clínico será de gran importancia, y la biopsia de vesículas seminales o la resonancia nuclear magnética son opciones recomendadas por algunos grupos, incluyendo la recomendación de otros de valorar el porcentaje de cilindros positivos y/o el porcentaje de tumor de cada cilindro.

\section{PROSTATECTOMÍA RADICAL + RADIOTERAPIA}

Una posible ventaja de proceder con PR en los tumores de alto riesgo sería la disminución de la incidencia de recidiva local. Algunos autores postulan que la exéresis radical de la próstata mejoraría el control local de la enfermedad, y reduciría el potencial de diseminación tardía de células de cáncer de próstata radioresistentes, si tuviera que realizarse adyuvancia posterior con RT, y simplificando el manejo de los niveles de PSA sérico en el seguimiento. La obtención del estadio patológico permitiría un seguimiento más fiel mediante mediciones seriadas de PSA, evitando la castración química inicial y todos sus indeseables efectos, a medio y largo término.

Es importante destacar que un tratamiento multimodal mediante PR con RT adyuvante, eliminaría potencialmente la enfermedad local, pero también aumentará la morbilidad asociada. Así, aunque algunas series destacan que la tasa de incontinencia no es más alta después de PR en cT3, existen estudios clínicos y anatómicos que sugieren que la incontinencia podría ser peor después de una amplia disección, que por otro lado es imprescindible en la cirugía de estos tumores. Esto es debido a que las bandeletas pueden contener fibras que aportan inervación al esfinter externo. Si a esta afectación le sumamos la probable fibrosis causada por la RT, la tasa de incontinencia o el retraso en la recuperación de la continencia, se verá aumentada comprometiendo la calidad de vida del paciente ${ }^{5,18,19}$. Existen datos que confirman una recuperación más rápi- 
da de la continencia después de una preservación de bandeletas neurovasculares uni o bilateralmente, pero en tumores de alto riesgo, la resección amplia y bilateral de bandeletas será obligada. Por esta misma razón, la preservación de la erección en estos pacientes sería de dudoso beneficio $^{20}$.

La HT neoadyuvante a la PR no ha demostrado mejorar las tasas de progresión bioquímica o clínica y supervivencia ${ }^{21-23}$. Pero la HT adyuvante si ha demostrado beneficios después de cirugía radical para el control de la enfermedad cT3, y es por esta razón que bastantes autores abogan por su uso precoz en cáncer de próstata pT3 de mal pronóstico ${ }^{6,12,24}$. También el uso de RT adyuvante después de la cirugía ha demostrado durante años disminuir el riesgo de recidiva local comparado con la PR en monoterapia, en pacientes de alto riesgo ${ }^{25-27}$. El estudio randomizado de la EORTC $22911^{28}$ demostró un aumento significativo de la supervivencia libre de enfermedad bioquímica y/o clínica en los pacientes con tumores pT2 con márgenes positivos o estadios pT3 que se trataron con RT adyuvante a PR, después de una mediana de seguimiento de 5 años. Incluso se observó beneficio en el subgrupo de pacientes con invasión de vesículas seminales. Si esto se traducirá en una ventaja de supervivencia total, todavía no está comprobado.

El tratamiento quirúrgico de los tumores de alto riesgo debe centrarse en una mayor extirpación radical que incluya, una extensa disección de ganglios linfáticos, una limpia exéresis del ápex prostático, e incluso una amplia resección bilateral de las bandeletas neurovasculares, con completa extirpación de las vesículas seminales, así como del cuello vesical. Una linfadenectomía limitada a los ganglios ilíacos internos y externos no será suficiente porque el cáncer de próstata ocurre preferentemente en el área caudal y periférica de la próstata, que drena a los linfáticos sacros laterales, y estos, a los del promontorio ${ }^{29}$. Por tanto, se ha propuesto que en los pacientes con alto riesgo de afectación ganglionar debe realizarse una extensa resección de los ganglios ilía$\cos$ internos ${ }^{30}$, permaneciendo en debate si existe beneficio de una disección más craneal hacia la bifurcación aórtica.

\section{RADIOTERAPIA}

A continuación se exponen una serie de consideraciones en referencia a la dosis de RT, técnica y extensión del tratamiento, así como su asociación a la HT.

\section{Dosis de radioterapia: aumento de dosis.}

Múltiples estudios (incluyendo aleatorizados) han demostrado el beneficio de la administración de dosis superiores a $70 \mathrm{~Gy}^{31-36}$. Si bien este beneficio se evidenció principalmente en el grupo de tumores de riesgo intermedio, los resultados actuales sugieren también un beneficio en el grupo de alto riesgo. En este sentido, el grupo del Memorial Sloan Kettering Cancer Center ha publicado recientemente los resultados obtenidos a 8 años en 561 pacientes tratados con dosis de 81 Gy utilizando técnicas de IMRT (RT de intensidad modulada): la supervivencia libre de recidiva bioquímica fue del $85 \%, 76 \%$ y $72 \%$ para el grupo de riesgo bajo, intermedio y alto respectivamente $^{37}$. Este mismo autor había publicado previamente un aumento de la supervivencia libre de recidiva bioquímica en el grupo de alto riesgo en función de la dosis administrada: $70 \%$ para dosis superiores a $81 \mathrm{~Gy}, 42 \%$ para dosis de 75,6 Gy y $21 \%$ en pacientes tratados con dosis inferiores a 75,6 $\mathrm{Gy}^{36}$. Otros estudios como el publicado por Valicenti ${ }^{34}$ han determinado una disminución de un $29 \%$ en la mortalidad por cáncer de próstata en el grupo de pacientes con tumores Gleason 8-10.

Los tumores de alto riesgo deben ser tratados con dosis altas probablemente $\geq 75$ Gy, sin conocerse cual es la dosis máxima por encima de la cual ya no se obtendría más beneficio. Las técnicas de braquiterapia (BQT) permiten administrar dosis elevadas superiores a las conseguidas con RT externa, y por lo tanto, parece obvio pensar que la mejor aumento de dosis podría obtenerse realizando un primer tiempo de tratamiento con RT externa incluyendo próstata y vesículas seminales ( \pm irradiación pélvica) y sobredosis con BQT de baja tasa con implante permanente (I- 125 o Pd -103) o de alta tasa con implante temporal con Ir-192. En este sentido, el grupo de Seattle ha publicado una supervivencia libre de recidiva bioquímica a 15 años del 67,8\% en el grupo de alto riesgo tratado con $\mathrm{RT}$ externa sobre pelvis $\mathrm{y}$ 
sobredosis prostática con I- $125^{38}$. El estudio multi-institucional fase II RTOG P-0019 diseñado para estimar la toxicidad de este tratamiento combinado determinó una toxicidad aguda grado 3 en 7,6\% de los pacientes y toxicidad tardía gastrointestinal y genitourinaria grado 3 en un $3,3 \%$. No se observó toxicidad grado 4 ó $5^{39}$.

\section{Irradiación electiva de las cadenas ganglionares pélvicas}

Los resultados derivados del ensayo de la RTOG $9413^{40}$ demuestran que la realización de RT pélvica electiva conjuntamente con la administración de HT aumenta la supervivencia libre de progresión en el grupo de pacientes con riesgo de afectación ganglionar superior al 15\%. Por otra parte, la realización de RTC-3D, y más aún, la aplicación de técnicas de IMRT en la irradiación pélvica están demostrando una mejor protección de los órganos de riesgo (vejiga, recto e intestino delgado) ${ }^{41}$.

\section{RADIOTERAPIA + HORMONOTERAPIA}

Existe una clara evidencia del beneficio de la asociación de la RT con HT adyuvante durante 23 años en los tumores de alto riesgo ${ }^{32,42-45}$. Todos estos estudios se han realizado con dosis de RT convencionales sin superar los 70 Gy y los resultados no son mejores que los obtenidos con RT exclusiva con aumento de dosis, lo cual podría dar lugar a la hipótesis de que la administración de altas dosis con las nuevas técnicas de RT, IMRT y IGRT (RT guiada por la imagen) son capaces de asegurar dosis plenas en el volumen tumoral puedan suplir el beneficio de la HT. En este sentido existen resultados iniciales derivados de estudios prospectivos que sugieren el beneficio de la utilización de la HT junto con la aumento de dosis sin aumentar la morbilidad de forma significativa ${ }^{46}$. También se han puesto en marcha estudios fase III que comparan tratamientos con altas dosis administradas con técnicas de IMRT versus dosis convencionales de 70,2 Gy (pero administradas con técnicas de IMRT) asociados a HT neoadyuvante y adyuvante de larga duración.

La realización de HT neoadyuvante a la RT estaría justificada si tenemos en cuenta los resultados derivados de estudios experimentales y clínicos que sugieren una reducción del volumen tumoral y potencialmente del número de células clonogénicas viables así como un aumento de la muerte celular programada o apoptosis si se administra de forma secuencial o concomitante. Estudios randomizados de la RTOG como el $86-10^{45,47}$ o $94-13^{40}$ demuestran mejores resultados en el grupo de pacientes con HT neoadyuvante y concomitante, traduciéndose en un aumento de la supervivencia libre de enfermedad bioquímica y clínica, así como la supervivencia causa específica. En el caso del estudio 86-10 pudo determinarse un aumento de la supervivencia global para el grupo de pacientes con Gleason 2-6. No se ha podido demostrar que la administración de más de 3 meses de HT neoadyuvante ofrezca mejores resultados ${ }^{48}$.

\section{OPCIONES FUTURAS DE TRATAMIENTO}

Independientemente del tratamiento realizado los resultados son poco satisfactorios.

La eficacia ya demostrada de la quimioterapia en la enfermedad diseminada ${ }^{49,50}$ ha dado lugar al diseño y realización de ensayos clínicos en que incorporan la administración de docetaxel, ya sea de forma concomitante con la RT (Grupo Español de Tratamiento de Tumores Génito Urinarios), neoadyuvante a la PR (experiencia de un grupo español en este sentido en tumores localmente avanzados), o bien una combinación de ambas. Los resultados son preliminares, pero en otras neoplasias es ya común el concepto de tratamiento neoadyuvante, por lo que en un tumor de mal pronóstico como el carcinoma de próstata localmente avanzado y con malos resultados con las terapias disponibles, parece un buen campo donde ensayar nuevas opciones terapéuticas ${ }^{51}$.

\section{CONCLUSIONES}

1. La monoterapia (PR, RT) no es suficiente para el tratamiento de los tumores de alto riesgo.

2. Se sugiere en algún estudio un mejor control local con cirugía en pacientes muy seleccionados.

3. El subgrupo de pacientes con cT3 con invasión microscópica capsular, Gleason menor de 8 y $\mathrm{PSA}<20 \mathrm{~g} / \mathrm{ml}$, podrían beneficiarse de un tratamiento quirúrgico así como los tumores localizados Gleason $\geq 8$. 
4. Los estadios pT2 con márgenes positivos y los estadios pT3 podrían beneficiarse de RT y/o HT adyuvante, en espera de resultados de ensayos clínicos que actualmente están en curso.

5. El tratamiento con RT debería incluir RTC3D pélvica y sobredosis prostática con dosis superiores a 70 Gy (probablemente como mínimo 75 Gy). BA de larga duración (2-3 años) iniciada 2-3 meses antes del inicio de la RT y mantenida durante y después de la misma.

6. El aumento de dosis podría realizarse con BQT después de irradiación pélvica en estos pacientes de alto riesgo para alcanzar dosis superiores a 75 Gy.

7. Las nuevas técnicas de radiación (IMRT, IGRT) pueden alcanzar dosis de $81 \mathrm{~Gy}$. A la espera de conocer la dosis óptima, éstas se han demostrado como las más eficaces hasta la actualidad en pacientes de alto riesgo.

8. Todavía no existen estudios aleatorizados que comparen la RTC-3D con las nuevas técnicas de irradiación (IMRT, IGRT).

Los pacientes con tumores de alto riesgo deberían incluirse en protocolos de investigación.

\section{REFERENCIAS}

1. Prostate cancer. In National Comprehensive Cancer Network (NCCN) Clinical PracticeGuidelines in Oncology, version 2.2005. Jenkinstown, PA: National Comprehensive Cancer Network, June 2006 (www.nccn).

2. Cooperberg MR, Moul JW, Carroll PR. The changing face of prostate cancer. J Clin Oncol. 2005 Nov 10;23(32):8146-8151.

3. Han M, Partin AW, Chan DY, Walsh PC. An evaluation of the decreasing incidence of positive surgical margins in a large retropubic prostatectomy series. J Urol. 2004 Jan;171(1):23-26.

4. Perez CA, Hanks GE, Leibel SA, Zietman AL, Fuks Z, Lee WR. Localized carcinoma of the prostate (stages T1B, T1C, T2, and T3) Review of management with external beam radiation therapy. Cancer. 1993 Dec 1;72(11):3156-3173.

5. Zietman AL, Coen JJ, Shipley WU, Willett CG, Efird JT. Radical radiation therapy in the management of prostate adenocarcinoma: The initial prostate specific antigen value as a predictor of treatment outcome. J Urol. 1994 Mar;151(3):640-645.

6. Aus G, Abbou CC, Bolla M, Heidenreich A, Schmid HP, van Poppel $\mathrm{H}$ et al. EAU guidelines on prostate cancer. Eur Urol. 2005 Oct;48(4):546-51.

7. Ward JF, Slezak JM, Blute ML, Bergstralh EJ, Zincke H. Radical prostatectomy for clinically advanced (cT3) prostate cancer since the advent of prostate-specific antigen testing: 15-year outcome. BJU Int. 2005Apr;95(6):751-756.

8. Carver BS, Bianco FJ Jr, Scardino PT, Eastham JA. Long-term outcome following radical prostatectomy in men with clinical stage T3 prostate cancer. J Urol. 2006 Aug; 176(2): 564-568.
19. Thompson IM Jr, Tangen CM, Paradelo J, Lucia MS, Miller G, Troyer D, et al. Adjuvant Radiotherapy for Pathologically Advanced Prostate Cancer: A Randomized Clinical Trial. JAMA. 2006 Nov 15;296(19):2329-2335.

10. Zincke H, Bergstralh EJ, Blute ML, Myers RP, Barrett DM, Lieber MM et al. Radical prostatectomy for clinically localized prostate cancer: long-term results of 1,143 patients from a single institution. J Clin Oncol. 1994 Nov; 12(11):2254-2263.

11. Berglund RK, Jones JS, Ulchaker JC, Fergany A, Gill I, Kaouk J, Klein EA. Radical prostatectomy as primary treatment modality for locally advanced prostate cancer: a prospective analysis. Urology. 2006 Jun;67(6):1253-1256. Epub 2006 May 6

12. Morgan WR, Bergstralh EJ, Zincke H. Long-term evaluation of radical prostatectomy as treatment for clinical stage C (T3) prostate cancer. Urology. $1993 \mathrm{Feb}$;1(2):113-120.

13. Van Poppel H, Goethuys H, Callewaert P, Vanuytsel L, Van de Voorde W, Baert L. Radical prostatectomy can provide a cure for well-selected clinical stage T3 prostate cancer. Eur Urol. 2000 Oct;38(4):372-379.

14. Kupelian PA, Buchsbaum JC, Elshaikh M, Reddy CA, Zippe C, Klein EA. Factors affecting recurrence rates after prostatectomy or radiotherapy in localized prostate carcinoma patients with biopsy Gleason score 8 or above. Cancer. 2002 Dec 1;95(11):2302-2307.

15. Van den Ouden D, Davidson PJ, Hop W, Schroder FH. Radical prostatectomy as a monotherapy for locally advanced (stage T3) prostate cancer. J Urol. 1994 Mar;151(3):646-651.

16. Witjes WP, Schulman CC, Debruyne FM. Preliminary results of a prospective randomized study comparing radical prostatectomy versus radical prostatectomy associated with neoadjuvant hormonal combination therapy in T2-3 N0 M0 prostatic carcinoma. The European Study Group on Neoadjuvant Treatment of Prostate Cancer. Urology. 1997 Mar;49(3A Suppl):65-69.

17. Villavicencio Mavrich H, Millán Rodríguez F, Chechile Toniolo G, Salinas Duffo D, Vicente Rodríguez J. Factores pronósticos y tablas predictivas del cáncer de próstata no localizado que excluirían la realización de una prostatectomía radical. Actas Urol Esp. 1998 Jul-Aug;22(7):581-591.

18. Karam I, Droupy S, Abd-Alsamad I, Korbage A, Uhl JF, Benoit G et al. The precise location and nature of the nerves to the male human urethra: histological and immunohistochemical studies with three-dimensional reconstruction. Eur Urol. 2005 Nov;48(5):858-64. Epub 2005 Mar 25.

19. Wei JT, Dunn RL, Marcovich R, Montie JE, Sanda MG. Prospective assessment of patient reported urinary continence after radical prostatectomy. J Urol. 2000 Sep;164(3 Pt 1):744748.

20. Stephenson RA, Middleton RG, Abbott TM. Wide excision (nonnerve sparing) radical retropubic prostatectomy using an initial perirectal dissection. J Urol. 1997 Jan;157(1): 251-255.

21. Gomella LG, Liberman SN, Mulholland SG, Petersen RO, Hyslop $\mathrm{T}$, Corn BW. Induction androgen deprivation plus prostatectomy for stage T3 disease: failure to achieve prostate-specific antigenbased freedom from disease status in a phase II trial. Urology. 1996 Jun;47(6):870-877.

22. Van Poppel H. Neoadjuvant hormone therapy and radical prostatectomy: the jury is still out. Eur Urol. 2001;39 Suppl 1:10-14.

23. Choo R, Hruby G, Hong J, Hong E, DeBoer G, Danjoux C, et al. Positive resection margin and/or pathologic T3 adenocarcinoma of prostate with undetectable postoperative prostate-specific antigen after radical prostatectomy: to irradiate or not? Int $J$ Radiat Oncol Biol Phys. 2002 Mar 1;52(3):674-680.

24. Lerner SE, Blute ML, Zincke H. Extended experience with radical prostatectomy for clinical stage T3 prostate cancer: outcome and contemporary morbidity. J Urol. 1995 Oct;154(4):1447-1452. 
25. Jacobson GM, Smith JA Jr, Stewart JR. Postoperative radiation therapy for pathologic stage C prostate cancer. Int J Radiat Oncol Biol Phys. 1987 Jul;13(7):1021-1014.

26. Bolla M, Van Poppel H, Van Caugh P, Vekemans K, Rigatti P, De Reijke $\mathrm{T}$ et al. Does postoperative radiotherapy after radical prostatectomy improve progression-free survival in pT3 N0 prostate cancer?. American Society of Clinical Oncology Annual Meeting proceedings 23:382, 2004.

27. Joniau S, Hsu CY, Lerut E, Van Baelen A, Haustermans K, Roskams T, Oyen R, Van Poppel H. A Pretreatment Table for the Prediction of Final Histopathology after Radical Prostatectomy in Clinical Unilateral T3a Prostate Eur Urol. 2007 Feb;51(2):388-94; discussion 395-6. Epub 2006 Jul 14.

28. Bolla M, van Poppel H, Collette L et al. Postoperative radiotherapy after radical prostatectomy: a randomised controlled trial (EORTC trial 22911). Lancet. 2005 Aug 13-19;366(9485):524-525.

29. Gil-Vernet JM. Prostate cancer: anatomical and surgical considerations. Br J Urol. 1996 Aug;78(2):161-168.

30. Bader P, Burkhard FC, Markwalder R, Studer UE. Is a limited lymph node dissection an adequate staging procedure for prostate cancer? J Urol. 2002 Aug; 168(2):514-518; discussion 518.

31. Bey P, Carrie C, Beckendorf V, Ginestet C, Aletti P, Madelis G et al. Dose escalation with 3D-CRT in prostate cancer: French study of dose escalation with conformal $3 \mathrm{D}$ radiotherapy in prostate cancer -Preliminary results. Int J Radiat Oncol Biol Phys. 2000 Sep 1;48(2):513-517.

32. Bey P, Carrie C, Beckendorf V, Ginestet C, Aletti P, Madelis G et al. Dose response in prostate cancer with 8-12 years follow-up. Int J Radiat Oncol Biol Phys 54:427-435, 2002.

33. Pollack A, Zagars GK, Starkschall G, Antolak JA, Lee JJ, Huang E, von Eschenbach AC, Kuban DA, Rosen I. Prostate cancer radiation dose response: results of the M.D. Anderson phase III randomized trial. Int $\mathrm{J}$ Radiat Oncol Biol Phys. 2002 Aug 1;53(5):1097-105.

34. Valicenti R, Lu J, Pilepich M, Asbell S, Grignon D. Survival advantage from higher-dose radiation therapy for clinically localized prostate cancer treated on the Radiation Therapy Oncology Group Trials. J Clin Oncol. 2000 Jul;18(14):2740-2746.

35. Zelefsky MJ, Fuks Z, Wolfe T, Kutcher GJ, Burman C, Ling CC et al. Locally advanced prostatic cancer: long-term toxicity outcomes after three-dimensional conformal radiation therapy -a dose escalation study. Radiology. 1998 Oct;209(1):169-174.

36. Zelefsky MJ, Fuks Z, Hunt M, Lee HJ, Lombardi D, Ling CC et al. High dose radiation delivered by intensity modulated conformal radiotherapy improves the outcome of localized prostate cancer. $\mathrm{J}$ Urol. 2001 Sep;166(3):876-881.

37. Zelefsky MJ, Chan H, Hunt M, Yamada Y, Shippy AM, Amols H Long-term outcome of high dose intensity modulated radiation therapy for patients with clinically localized prostate cancer. J Urol. 2006 Oct;176(4 Pt 1):1415-1419.

38. Sylvester JE, Grimm PD, Blasko JC, Millar J, Orio PF 3rd, Skoglund $\mathrm{S}$ et al. 15-Year biochemical relapse free survival in clinical Stage T1-T3 prostate cancer following combined exter nal beam radiotherapy and brachytherapy; Seattle experience. Int J Radiat Oncol Biol Phys. 2007 Jan 1;67(1):57-64. Epub 2006 Nov 2.

39. Lee WR, DeSilvio M, Lawton C, Gillin M, Morton G, Firat S et al. A phase II study of external beam radiotherapy combined with permanent source brachytherapy for intermediate-risk, clinically localized adenocarcinoma of the prostate: preliminary results of RTOG P-0019. Int J Radiat Oncol Biol Phys. 2006 Mar 1;64(3):804-809. Epub 2005 Nov 14.

40. Roach M 3rd, DeSilvio M, Lawton C, Uhl V, Machtay M, Seider MJ et al. Phase III trial comparing whole-pelvic versus prostate-only radiotherapy and neoadjuvant versus adjuvant combined androgen suppression: Radiation Therapy Oncology Group 9413. J Clin Oncol. 2003 May 15;21(10):1904-1911.

41. Wang-Chesebro A, Xia P, Coleman J, Akazawa C, Roach M 3rd. Intensity-modulated radiotherapy improves lymph node coverage and dose to critical structures compared with three-dimensional conformal radiation therapy in clinically localized prostate cancer. Int J Radiat Oncol Biol Phys. 2006 Nov 1;66(3):654-662.

42. Bolla M, Collette L, Blank L, Warde P, Dubois JB, Mirimanoff RO et al. Long-term results with immediate androgen suppression and external irradiation in patients with locally advanced prostate cancer (an EORTC study): a phase III randomised trial. Lancet. 2002 Jul 13;360(9327):103-106.

43. Roach M 3RD, Lu J, Pilepich MV, Asbell SO, Mohiuddin M, Terry $\mathrm{R}$ et al. Predicting long-term survival, and the need for hormonal therapy: a meta-analysis of RTOG prostate cancer trials. Int $\mathrm{J}$ Radiat Oncol Biol Phys. 2000 Jun 1;47(3):617-627.

44. Hanks GE, Pajak TF, Porter A, Grignon D, Brereton H, Venkatesan V et al. Phase III trial of long-term adjuvant androgen deprivation after neoadjuvant cytoreduction and radiotherapy in locally advanced carcinoma of the prostate: The Radiation Therapy Oncology Group protocol 92-02. J Clin Oncol. 2003 Nov 1;21(21):3972-3978.

45. Pilepich MV, Winter K, Lawton CA, Krisch RE, Wolkov HB, Movsas $\mathrm{B}$ et al. Androgen suppression adjuvant to definitive radiotherapy in prostate carcinoma-long-term results of phase III RTOG 8531. Int J Radiat Oncol Biol Phys. 2005 Apr 1;61(5):1285-1290.

46. Zapatero A, Valcarcel F, Calvo FA, Algas R, Bejar A, Maldonado J et al. Risk-adapted androgen deprivation and escalated threedimensional conformal radiotherapy for prostate cancer: Does radiation dose influence outcome of patients treated with adjuvant androgen deprivation? A GICOR study. J Clin Oncol. 2005 Sep 20;23(27):6561-6568.

47. Pilepich MV, Winter K, John MJ, Mesic JB, Sause W, Rubin P et al. Phase III Radiation Therapy Oncology Group (RTOG) trial 8610 of androgen deprivation adjuvant to definitive radiotherapy in locally advanced carcinoma of prostate. Int $\mathrm{J}$ Radiat Oncol Biol Phys. 2001 Aug 1;50(5):1243-1252.

48. Crook J, Ludgate C, Malone S, Lim J, Perry G, Eapen L et al. Report of a multicenter Canadian phase III randomized trial of 3 months vs. 8 months neoadjuvant androgen deprivation before standard-dose radiotherapy for clinically localized prostate cancer. Int J Radiat Oncol Biol Phys. 2004 Sep 1;60(1):15-23.

49. Petrylak DP, Tangen CM, Hussain MH, Lara PN Jr, Jones JA, Taplin ME et al. Docetaxel and estramustine compared with mitoxantone and prednisone for advanced refractory prostate cancer. N Engl J Med. 2004 Oct 7;351(15):1513-1520

50. Tannock IF, de Wit R, Berry WR, et al. Docetaxel plus prednisone or mitoxantrone plus prednisone for advanced prostate cancer. $\mathrm{N}$ Engl J Med. 2004 Oct 7;351(15):1502-1512.

51. Eisenberger MA. A multi-institutional pilot study of adjuvant docetaxel for patients with prostate cancer at high risk for relapse after radical prostatectomy. Rev Urol. 2003;5 Suppl 2:S42-47.

Correspondencia autor: Dr. S. Esquena

Servicio de Urología. Fundación Puigvert

Cartagena, 340-350. 08025 Barcelona

Tel.: 934169700

E-mail autor: dr.esquena@gmail.com

Información artículo: Revisión de Conjunto - Cáncer de próstata

Trabajo recibido: febrero 2007

Trabajo aceptado: abril 2007 